\title{
Long-term effects of a partially supervised conditioning programme in cystic fibrosis
}

\author{
H. Hebestreit*, S. Kieser*, S. Junge \#, M. Ballmann\#, A. Hebestreit*, C. Schindler", \\ T. Schenk ${ }^{\star}$, H-G. Posselt ${ }^{+}$and S. Kriemler ${ }^{\S}$
}

ABSTRACT: Little is known about the long-term persistence of positive effects induced by a physical conditioning programme in cystic fibrosis. Therefore, this study determined the effects of a 6-month conditioning programme on peak oxygen uptake (primary outcome) and other markers of fitness, physical activity, anthropometry, lung function and quality of life (secondary outcomes), 18 and 24 months after the programme was initiated.

Patients with cystic fibrosis aged 12-40 yrs were randomly assigned to an intervention $(n=23)$ and a control $(n=15)$ group. The intervention group consented to add $3 \mathrm{~h}$ of sports per week for $\geqslant 6$ months to their previous activities. Controls were asked to maintain their level of activity for 12 months. Patients were seen at baseline and after 3, 6, 12, 18 and 24 months.

There was no significant difference between groups at baseline. The intervention induced positive effects on peak oxygen uptake (difference in changes from baseline to the 18- and 24month assessments between groups: $3.72 \pm 1.23 \mathrm{~mL} \cdot \mathrm{min}^{-1} \cdot \mathrm{kg}^{-1} ; \mathrm{p}<0.01$ ), maximal workload $\left(0.37 \pm 0.11 \mathrm{~W} \cdot \mathrm{kg}^{-1} ; \mathrm{p}<0.01\right)$, vigorous physical activity $\left(1.63 \pm 0.82 \mathrm{~h} \cdot\right.$ week $\left.^{-1} ; \mathrm{p}<0.05\right)$, forced vital capacity $(6.06 \pm 2.87 \%$ predicted; $p<0.05)$ and perceived health $(9.89 \pm 4.72 ; p<0.05)$.

A home-based partially supervised physical conditioning programme can improve physical fitness, lung function and perceived health long after the intervention has ended.

KEYWORDS: Cystic fibrosis, exercise capacity, lung function, pulmonary rehabilitation, quality of life

everal studies have demonstrated favourable effects of exercise programmes in children and adults with cystic fibrosis [15]. Specifically, an increase in physical activity was associated with a gain in exercise capacity $[1$, $3,5]$, a stabilisation or even an improvement in lung function $[1,2,4]$ and a higher quality of life $[1,5]$. However, it is unclear whether the benefits induced by an exercise programme are maintained for a time period longer than 3 months.

Supervised conditioning programmes $[1,3,5]$ and home-based approaches $[2,4]$ were both effective at inducing positive health effects in patients with cystic fibrosis. Yet, a home-based individualised conditioning programme has several conceivable advantages: 1) the programme can be easily implemented; 2) the patients can choose activities based on their personal preferences; 3) the accessibility of the activities in respect to location and time is much greater; and 4) activities can be planned together with family members or friends. Therefore, we hypothesised that activities initiated as part of a home-based programme might be maintained beyond the end of the programme.

Peak oxygen uptake $\left(V^{\prime} \mathrm{O}_{2}\right.$,peak $)$ has been used as a marker of aerobic fitness and physical activity [6]. In cystic fibrosis, $V^{\prime} \mathrm{O}_{2}$,peak has been shown to be related to physical activity [7] and responded to physical conditioning within 2 weeks of an intense inpatient programme [1], or within 3 months of a supervised outpatient programme [3]. Furthermore, $V^{\prime} \mathrm{O}_{2}$,peak seems to be an important predictor of cystic fibrosis-related mortality [8].

The primary objective of this randomised controlled trial was to determine the effects of a home-based, individualised, partially supervised conditioning programme on $V^{\prime} \mathrm{O}_{2}$, peak, $12-$ 18 months after the programme had ended. Secondary outcome measures included other markers of exercise capacity, physical activity, body composition, lung function and quality of life (QoL).
AFFILIATIONS

*Paediatric Dept, Julius-

Maximilians-Universität Würzburg, Würzburg,

\#Paediatric Pulmonology and Neonatology, Medizinische Hochschule, Hanover, and +Paediatric Dept, Johann Wolfgang Goethe Universität, Frankfurt, Germany.

"Institute of Social and Preventive

Medicine, Basel, and

${ }^{\S}$ Institute of Exercise and Health

Sciences, Basel, Switzerland

CORRESPONDENCE

H. Hebestreit

Universitäts-Kinderklinik Josef-Schneider-Str. 2 97080 Würzburg Germany

E-mail: hebestreit@

uni-wuerzburg.de

Received:

April 142009

Accepted after revision:

July 232009

First published online:

July 302009 


\section{METHODS}

Details on the sample size justification, the randomisation procedure and the study's design and procedures are provided in an online supplement.

\section{Subjects}

Patients with cystic fibrosis were recruited from the German cystic fibrosis centres at Frankfurt, Hanover and Würzburg. Inclusion criteria for this study were a confirmed diagnosis of cystic fibrosis (i.e. a typical clinical picture and at least two positive sweat tests or cystic fibrosis-relevant mutations on both alleles of the CFTR gene), an age of $\geqslant 12 \mathrm{yrs}$, a forced expiratory volume in $1 \mathrm{~s}$ (FEV1) of $\geqslant 35 \%$ of predicted [9] and the ability to perform physical activity. Exclusion criteria were non-cystic fibrosis-related chronic diseases and cystic fibrosisrelated conditions posing an increased risk to the patient when exercising. These were specifically oesophageal varicosis, pulmonary bullae, a $<80 \%$ drop in arterial oxygen saturation with exercise and signs of pulmonary hypertension on electrocardiogram and/or echocardiogram.

40 patients agreed to participate. Written informed consent was obtained from the patients and their guardians, if the patients were under 18 yrs of age. The ethics committees of the medical faculties of all participating centres (Frankfurt, Hanover and Würzburg) approved the study protocol and procedures.

Figure 1 summarises the flow of patients through the study. Two patients were excluded from the study at the baseline visit due to a FEV1 below $35 \%$ predicted. No patients were excluded for any of the other specified exclusion criteria.
At the end of the baseline assessments, patients were randomly assigned to the intervention or the control group. The procedure allocated $60 \%$ of all patients to the intervention group since we expected more dropouts in this group. In the process of the study, five patients decided to discontinue their participation. One male control patient moved away and was not willing to travel; one female patient in the intervention group decided to join another study. Reasons were not given for the other dropouts.

Owing to exacerbations, some patients could not be tested on all scheduled visits. During the time period between baseline and 6 months, 22 patients in the intervention group and all 15 patients in the control group were seen at least once. The number of patients investigated at 12 months was 19 in the intervention group and 12 in the control group. During the 18to 24-month period, 20 patients in the intervention group and 13 in the control group were assessed at least once.

\section{Study design and procedures}

Patients were seen in their respective centres at baseline, and after 3, 6, 12, 18 and 24 months. At each visit, $V^{\prime} \mathrm{O}_{2}$,peak (primary outcome) was determined as highest $V^{\prime} \mathrm{O}_{2}$, over $30 \mathrm{~s}$ during a continuous incremental cycling task to volitional fatigue [10]. Maximum work ( $W$ max) was taken as the power maintained over the final completed 1-min stage. A Wingate test was employed to determine muscle power (peak power (PP) and mean power (MP)) [11]. Engagement in vigorous physical activity was determined by accelerometry over 7 days. Height and body mass were measured and skinfold thickness was determined at four sites. Percentage body fat was calculated using age-specific equations [12, 13]. FEV1,

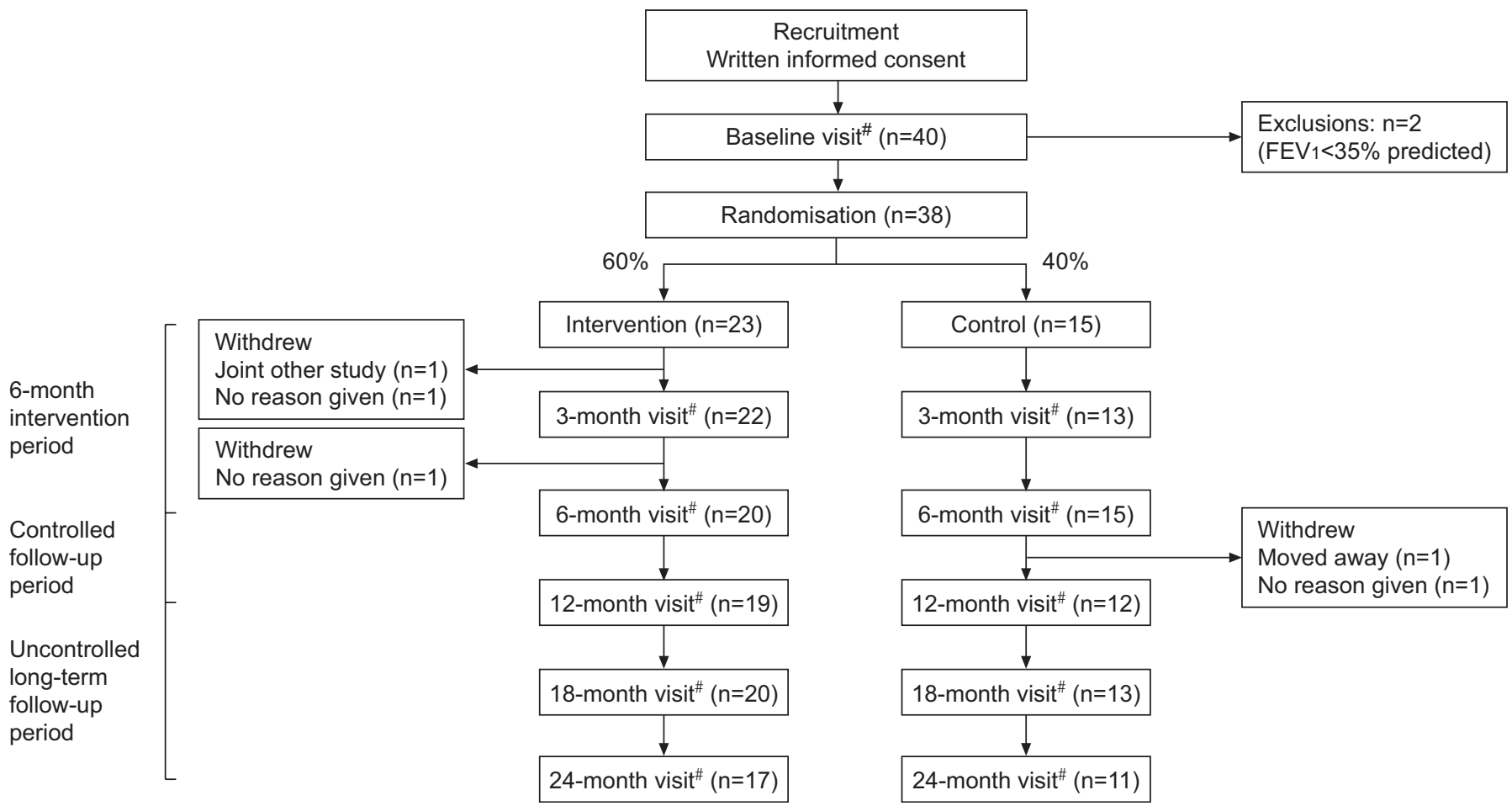

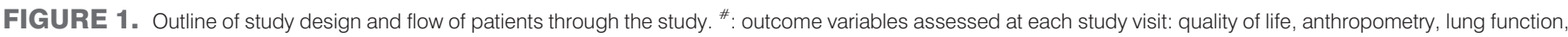
muscle power, maximal aerobic power, peak oxygen uptake, physical activity. FEV1: forced expiratory volume in $1 \mathrm{~s}$. 
forced vital capacity (FVC) and residual volume relative to total lung capacity (RV/TLC) were determined and expressed as $\%$ predicted $[9,14,15]$. QoL was assessed by questionnaire (CFQ-14+) [16].

\section{Intervention}

Patients in the intervention group consented to increase their sport activities by a minimum of $3 \times 60 \mathrm{~min} \cdot \mathrm{week}^{-1}$ for the first 6 months of the study. All patients in the intervention group received activity counselling at the baseline visit and at visits after 3 and 6 months. First, an exercise specialist and the patient discussed options about incorporating intense physical activities in daily life. Based on the results of the exercise tests and clinical data, an individual activity plan was generated which incorporated the patients' preferred physical activities and availability near their homes. Patients were equipped with a heart rate monitor (Accurex plus, Polar Electro, Büttelbron, Germany) and provided with a target heart rate for endurance-type activities that was just below the heart rate at the individual gas exchange threshold [17]. Patients were also encouraged to perform strength-enhancing exercises, since strength-building activities and interval training have been shown to be effective for conditioning and improving health in cystic fibrosis patients [18].

Four of the 23 patients in the intervention group chose to engage exclusively in endurance-type sports (cycling, jogging, swimming) whereas three chose ball games (squash, badminton, football). Five patients combined weight training with endurance training in a fitness centre, and the remaining 11 patients of the intervention group engaged in a variety of activities combining endurance-type sports with ball games and strengthening exercises.

Logistic support and, if needed, financial help (maximum of $€ 200$ ) was offered to the patients to foster the realisation of the activity plan. For example, the specialist called the director of a fitness centre in order to facilitate admission of a patient and to stimulate specific supervision and guidance. Alternatively, the patient received financial support to become a member of a sports club. Patients in the intervention group were called several times during the first 6 months of the study to check on their activity behaviour and, if necessary, to offer additional help. After the first 6 months, patients in the intervention group were encouraged to maintain or further increase their activity level.

Patients in the control group were told to keep their physical activity level constant for the first 12 months of the study. Thus, the assessment at 12 months marked the end of the controlled follow-up period. During the second year of the study, the open follow-up period, control subjects were free to change their physical activity behaviour.

\section{Data analysis and statistics}

The intervention and control groups were compared for baseline characteristics using an unpaired t-test for continuous and Chisquared statistics for dichotomous data. Groups were compared for the incidence of reported febrile infections at each visit using Chi-squared statistics. The rank sum test was used to assess group differences in attitude towards sports activities at each visit and changes from baseline were analysed separately for each group using the matched signed rank test.
For primary and secondary outcome variables, changes from baseline to subsequent visits were calculated. To assess the long-term effects of the intervention, the changes from baseline to 18 months and 24 months were compared between groups using ANOVA for unbalanced repeated measures allowing for missing data. In case of a significant group difference, two subsequent analogous tests were performed: for the main intervention period up to 6 months, and for the 12-month visit. Estimates of the effects of the intervention and their standard errors were obtained from the ANOVA analyses.

All analyses were performed using BMDP statistical software release 7 (Statistical Solutions, Cork, Ireland). A p-value $<0.05$ was considered significant. Data are presented as mean $\pm \mathrm{SD}$, unless otherwise stated.

\section{RESULTS}

Physical characteristics and activity behaviour of the study population are summarised in table 1 . At baseline, there were no significant differences between the groups. There was also no difference between the groups in any QoL domain (data not shown).

Relative to baseline and the control group, the intervention group reported a significant increase in sports activities of $2.16 \pm 0.49 \mathrm{~h} \cdot$ week $^{-1}$ during the first 6 months of the study. For the same time period, the increase in vigorous physical activity in the intervention group measured objectively by accelerometry was $1.05 \pm 0.43 \mathrm{~h} \cdot$ week $^{-1}$ (nonsignificant). There was no difference between the groups in the reported number of infections with fever at any time during the study. There was

\begin{tabular}{|c|c|c|}
\hline & $\begin{array}{l}\text { Intervention } \\
\text { group }\end{array}$ & $\begin{array}{l}\text { Control } \\
\text { group }\end{array}$ \\
\hline Subjects & 23 & 15 \\
\hline Male & 13 & 6 \\
\hline Female & 10 & 9 \\
\hline Age yrs & $19.5 \pm 6.4$ & $19.4 \pm 5.3$ \\
\hline Height $\mathrm{cm}$ & $165.6 \pm 11.6$ & $165.7 \pm 11.3$ \\
\hline Body mass kg & $55.5 \pm 13.7$ & $56.8 \pm 13.3$ \\
\hline $\begin{array}{l}\text { Sum of four skinfold measurements } \\
\mathrm{mm}\end{array}$ & $37.4 \pm 13.9$ & $35.3 \pm 12.1$ \\
\hline Body fat \% & $17.8 \pm 7.4$ & $17.2 \pm 6.5$ \\
\hline Lean body mass $\mathbf{k g}$ & $45.3 \pm 11.0$ & $47.0 \pm 11.7$ \\
\hline FVC \% pred & $81.5 \pm 13.1$ & $88.2 \pm 16.3$ \\
\hline FEV $_{1} \%$ pred & $69.1 \pm 17.2$ & $75.6 \pm 21.9$ \\
\hline RV/TLC \% pred & $172.1 \pm 46.8$ & $161.6 \pm 68.3$ \\
\hline Vigorous physical activity $h \cdot$ week $^{-1}$ & $4.31 \pm 2.68$ & $6.27 \pm 2.93$ \\
\hline$V^{\prime} \mathrm{O}_{2}$, peak $\mathrm{mL} \cdot \mathrm{min}^{-1} \cdot \mathbf{k g}^{-1}$ & $37.2 \pm 8.9$ & $39.4 \pm 6.9$ \\
\hline$W \max \mathbf{W} \cdot \mathbf{k g}^{-1}$ & $2.81 \pm 0.58$ & $2.98 \pm 0.62$ \\
\hline PP $\mathbf{W} \cdot \mathbf{k g}^{-1}$ & $9.50 \pm 1.51$ & $8.89 \pm 1.63$ \\
\hline MP $\mathbf{W} \cdot \mathbf{k g}^{-1}$ & $6.59 \pm 1.19$ & $6.38 \pm 1.16$ \\
\hline
\end{tabular}

Data are presented as $n$ or mean \pm SD. FVC: forced vital capacity; \% pred: \% predicted; FEV1: forced expiratory volume in $1 \mathrm{~s}$; RV: residual volume; TLC: total lung capacity; $V^{\prime} \mathrm{O}_{2}$,peak: peak oxygen uptake; $W_{\text {max: }}$ power maintained over the final completed 1-min stage; PP: peak power; MP: mean power. 
also no difference between the groups in the reported attitude towards sports activities at baseline and during the follow-up visits and no change in the reported attitude over time.

\section{Primary outcome}

Figure 2 shows the effect of the intervention on the primary outcome variable $V^{\prime} \mathrm{O}_{2}$, peak. There was a significant intervention effect on the change in $V^{\prime} \mathrm{O}_{2}$,peak during the long-term, open follow-up period, 12-18 months after the 6-month intervention (table 2). Subsequent analyses showed significant group differences for the main 6-month training period, but not for the assessment at 12 months (table 2).

\section{Secondary outcomes}

Results of secondary outcomes with significant effects of the intervention at 18-24 months are documented in table 2.

\section{Work capacity}

A significant effect was observed for $W \max$ at long-term followup and during the initial 6-month training period (table 2).

\section{Muscle power}

There was no effect of the intervention on PP and MP at 1824 months.

\section{Physical activity}

The change in time spent in vigorous activities as assessed by accelerometry was significantly different between the groups during the open, long-term follow-up period (table 2). Although there was a trend for vigorous activities in favour of the intervention group throughout the initial 12 months of the study, the effect was not significant.

\section{Anthropometry}

There was no effect of the intervention on changes in either height or body mass. However, a significant group difference in the change in skinfold thickness occurred between baseline and the assessments at 18-24 months (table 2). Relative to the control group, the intervention group experienced a decrease in skinfold thickness, which was also evident at the 12-month

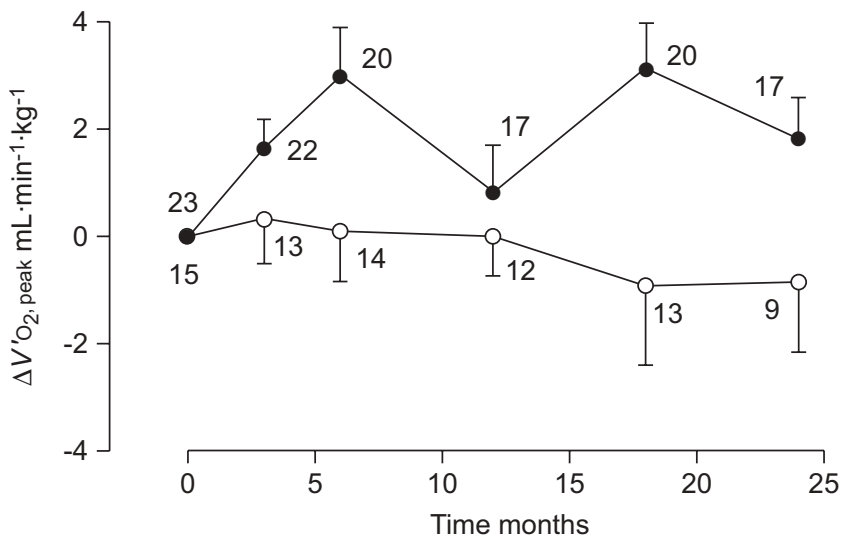

FIGURE 2. Change in peak oxygen uptake $\left(V^{\prime} \mathrm{O}_{2}\right.$,peak $)$ from baseline in the intervention and the control groups. Data are presented as mean $\pm \mathrm{SE}$ with the number of patients indicated. There was a significant difference between groups $(p<0.01)$ and a significant group $\times$ time interaction $(p<0.01)$ in the repeated measures ANOVA involving all five follow-up time points. $\bullet$ : intervention group; $\bigcirc$ : control group. visit. There was no significant difference between the groups in the change in percentage body fat or lean body mass.

\section{Lung function}

As shown in table 2, the difference in FVC change from baseline to 18-24 months between groups was about 6\% predicted favouring the intervention group, while no differences between groups were observed during the first year of the study. No effects of the intervention were evident for FEV1 and RV/TLC.

\section{Quality of life}

There was no significant effect of the intervention on QoL scales except for the scale "Subjective health perception". Here, the intervention group profited during the 18- to 24-month follow-up and the main 6-month intervention period (table 2).

\section{DISCUSSION}

This study documents that positive health effects of a 6-month home-based, partially supervised conditioning programme can be observed 12 months and more after the end of the intervention. These favourable long-term health effects were obtained for aerobic fitness, vigorous physical activity and FVC, which are important and relevant determinants of health in cystic fibrosis $[8,19]$. Furthermore, the patients' subjective health perception remained positively affected by the intervention long after the programme had ended.

The difference in change of aerobic fitness (i.e. $V^{\prime} \mathrm{O}_{2}$,peak and $W$ max) between the intervention and control group at longterm follow-up was about $10 \%$ of baseline values. This positive effect was reached right after the 6-month, partially supervised intervention period but, more importantly, was also evident at long-term follow-up without any further formal intervention and supervision. This novel finding is promising and shows that a 6-month, partially supervised and individualised physical activity programme might suffice to induce persisting changes in activity behaviour and health. This increase is clinically relevant when considering that $V^{\prime} \mathrm{O}_{2}$,peak is an important predictor of mortality [8].

Surprisingly, no effect of the intervention on $V^{\prime} \mathrm{O}_{2}$,peak and Wmax was evident at the 12-month visit, despite significant effects during the 6-month training period and at the long-term follow-up after 18-24 months (fig. 2 and table 2). This phenomenon is not easily explained. It does not reflect differences in sample composition among time periods since an analysis including only patients with complete data revealed the same pattern. A potential reason might be that the 12-month assessments took place during winter when most health problems occur [20]. Although there was no difference between the groups in the reported number of febrile infections between the 6-month and 12-month assessment, subclinical exacerbations inducing a transient reduction in vigorous activities might have occurred in the intervention group reducing the patients' physical activity. This hypothesis is in line with the intervention group's perception of health, which was lowest at the 12-month visit. Another possible explanation for the nonsignificant effect of the intervention on aerobic fitness at 12 months could be an intermittent decline in exercise adherence because of the cold winter season.

Skinfold thickness decreased more in the intervention group compared with the control group. At first, this seems alarming 


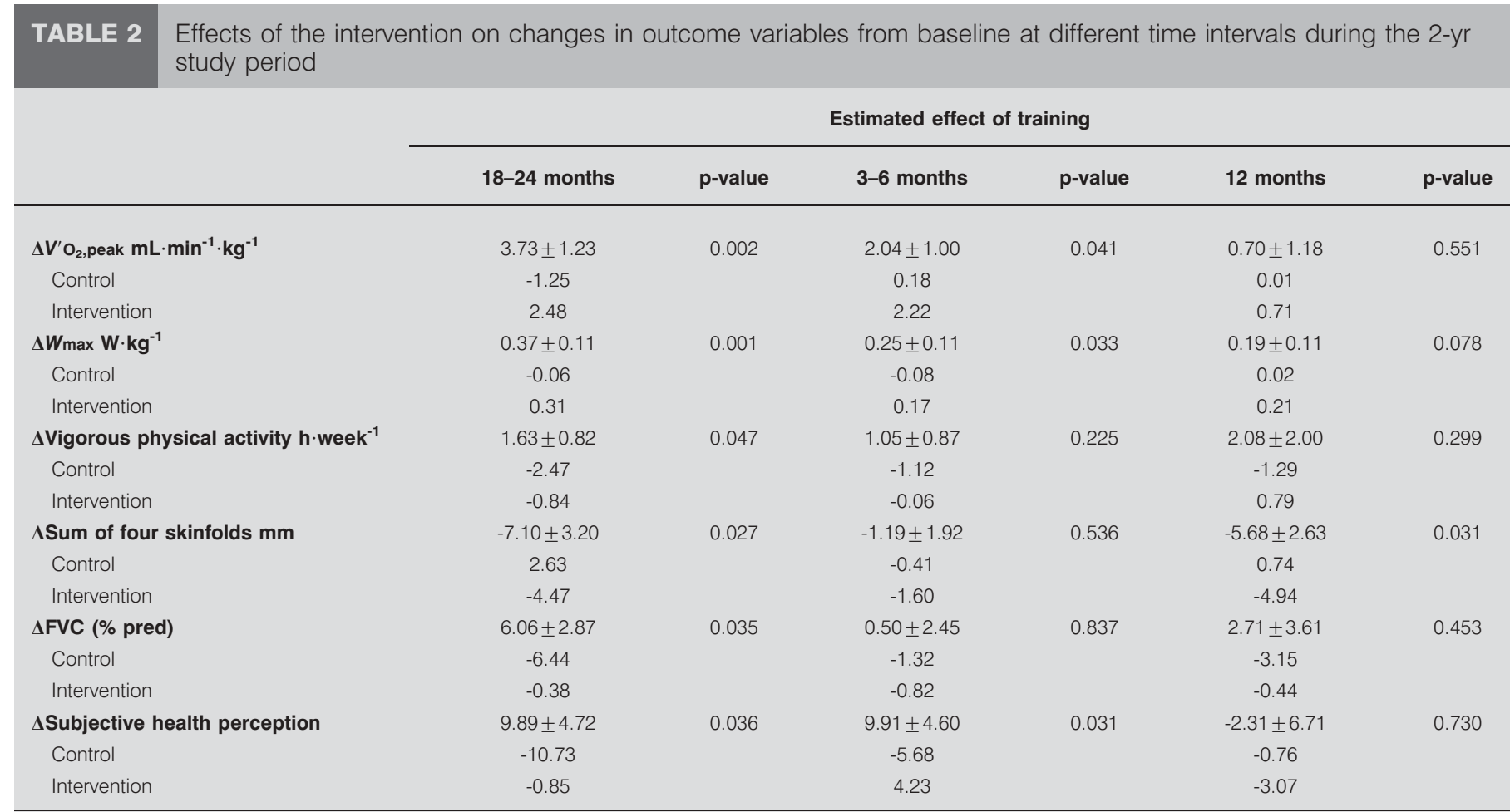

Data are presented as mean \pm SE or mean, unless otherwise stated. $\Delta V^{\prime} \mathrm{O}_{2}$,peak: change in peak oxygen uptake from baseline; $\Delta W$ max: change in maximal workload during an incremental cycling task; $\triangle$ FVC: change in forced vital capacity from baseline, \% pred: \% predicted. The reported effects are estimated regression coefficients and their asymptotic standard errors. Positive values indicate that the intervention group had a larger increase or a smaller decrease in the respective parameter than the control group. Mean changes from baseline for the control group and the intervention group are also given for each time period in the study

since a poor nutritional status has been associated with higher mortality in cystic fibrosis patients [21]. However, there were no differences in changes of height or weight between the groups and no significant effect of the intervention on percentage body fat. A significant increase in muscle mass was not reached, possibly because the intervention was rather mild.

In line with other studies evaluating the effects of a conditioning programme in cystic fibrosis lasting $\geqslant 6$ months, we found positive effects of the intervention on FVC but not on FEV1 [2,4]. Our data suggest that the beneficial effect on FVC is not caused by a decrease in pulmonary hyperinflation since no effect of the intervention on RV/TLC was observed. We can only speculate that the regular exercise might have resulted in a concomitant training of respiratory muscles, thereby increasing FVC [22-25].

Concordant with the significant long-term effects of the intervention on aerobic fitness and lung function, patients in the intervention group perceived significant and long-lasting benefits to their health compared with control patients. However, no effect on any other scale of the QoL questionnaire was demonstrable. This is somewhat surprising since traininginduced changes in physical fitness have been associated with changes in the physical functioning scale, another QoL domain assessed with the cystic fibrosis questionnaire used in the present study [5]. We can only speculate that factors other than physical fitness affect the QoL scales in such a way that the effects of increased aerobic capacity were clouded in the present study.
For long-term benefits, an intervention programme involving physical conditioning should be able to modify exercise behaviour towards an active lifestyle. The long-lasting effects of the intervention observed in the current study suggest that a partially supervised, individualised, home-based approach may be sufficient to change activity behaviour in patients with cystic fibrosis. The lasting effects of a 6-month home-based conditioning programme are of major clinical importance since such a programme can be realised relatively easily. The practical implementation of such a programme requires the assessment of exercise-related risks based on the patient's medical status, his or her preferred physical activities and, in some patients, the results of an exercise test to rule out exercise-induced hypoxaemia or arrhythmia [26, 27]. With respect to beneficial effects, the type of activity chosen by the patient for his or her exercise routine probably does not matter. At least in the short term, anaerobic [5] and aerobic exercises [1-3], as well as strength training [1] or a combination thereof [4], have been effective in enhancing fitness and health in cystic fibrosis patients.

There are some limitations of this study to be addressed. First, although not statistically significant, the control group appeared somewhat healthier and more active at baseline than the intervention group (table 1). We cannot exclude that this difference might have favoured a positive change in healthrelated outcomes in the intervention group that was not related to the intervention itself. However, there is some evidence that patients who did not do well in the past might have a faster decline of health status in the future [28]. Thus, the somewhat 
better health status of the controls in our study at baseline could also strengthen our results. Secondly, there might have been a bias towards positive effects of the intervention for $V^{\prime} \mathrm{O}_{2}$,peak and skinfold measurements since the outcome assessors were not blinded for these parameters. However, great care was taken that each individual measurement was administered following standardised procedures and criteria and, in case of the exercise tests, was performed to exhaustion irrespective of group allocation. Thirdly, engagement in vigorous activities was, on average, not increased by the intended extent (2.16 instead of $3 \mathrm{~h} \cdot$ week $^{-1}$ ), which might reflect under-reporting or, most likely, insufficient compliance with the intervention. Yet, the effects of the programme were still significant in favour of the intervention. Finally, the incomplete long-term patient follow-up, an inherent feature in 2-yr-long intervention studies, might somewhat compromise the validity of the results. However, the percentage of patients tested at each scheduled assessment was similar in the intervention and control groups (fig. 1) and we were able to assess $>85 \%$ of all patients recruited at least once in the 18- to $24-$ month follow-up period.

In conclusion, a 6-month home-based partially supervised and individualised physical conditioning programme led to longlasting beneficial effects on physical fitness, activity and lung function in patients with cystic fibrosis $>1 \mathrm{yr}$ after the intervention had ended. This finding indicates that persisting changes in activity behaviour can be initiated by such a programme.

\section{SUPPORT STATEMENT}

This study was supported by a grant from the German cystic fibrosis foundation (Mukoviszidose e.V.)

\section{CLINICAL TRIALS}

This study is registered as clinical trial at www.clinicaltrials.gov (NCT00231686)

\section{STATEMENT OF INTEREST}

None declared.

\section{ACKNOWLEDGEMENTS}

The authors would like to thank C. Smaczny, M. Heilmann (both Pulmonology, Medizinische Hochschule, Hanover, Germany), J. Bargon (Pulmonology and Allergology, Johann Wolfgang Goethe Universität, Frankfurt, Germany) and H. Stobbe (Pediatric Pulmonology and Neonatology, Medizinische Hochschule, Hanover) for their support in recruiting patients and conducting the assessments.

\section{REFERENCES}

1 Selvadurai HC, Blimkie CJ, Meyers N, et al. Randomized controlled study of in-hospital exercise training programs in children with cystic fibrosis. Pediatr Pulmonol 2002; 33: 194-200.

2 Schneiderman-Walker J, Pollock SL, Corey M, et al. A randomized controlled trial of a three year home exercise program in cystic fibrosis. J Pediatr 2000; 136: 304-310.

3 Orenstein DM, Franklin BA, Doerchuk CF, et al. Exercise conditioning and cardiopulmonary fitness in cystic fibrosis. The effects of a three-month supervised running program. Chest 1981; 80: 392-398.

4 Moorcroft AJ, Dodd ME, Morris J, et al. Individualized unsupervised exercise training in adults with cystic fibrosis: a 1 year randomized controlled trial. Thorax 2004; 59: 1074-1080.

5 Klijn PHC, Oushoorn A, van der Ent CK, et al. Effects of anaerobic training in children with cystic fibrosis: a randomized controlled study. Chest 2004; 125: 1299-1305.
6 US Department of Health and Human Services. Physical activity and health: a report of the Surgeon General. Atlanta, Centers for Disease Control and Prevention, 1996.

7 Hebestreit H, Kieser S, Rüdiger S, et al. Physical activity is independently related to aerobic capacity in cystic fibrosis. Eur Respir J 2006; 28: 734-739.

8 Nixon PA, Orenstein DM, Kelsey SF, et al. The prognostic value of exercise testing in patients with cystic fibrosis. N Engl J Med 1992; 327: $1785-1788$.

9 Sherrill DL, Lebowitz MD, Knudson RJ, et al. Continuous longitudinal regression equations for pulmonary function measures. Eur Respir J 1992; 5: 452-462.

10 Godfrey S, Davies CTM, Wozniak E, et al. Cardio-respiratory response to exercise in normal children. Clin Sci 1971; 40: 419-431.

11 Inbar O, Bar-Or O, Skinner JS. The Wingate Anaerobic Test Champaign, Human Kinetics, 1996.

12 Slaughter MH, Lohman TG, Boileau RA, et al. Skinfold equations for estimation of body fatness in children and youth. Hum Biol 1988; 60: 709-723.

13 Durnin JVGA, Womersley J. Body fat assessed from total body density and its estimation from skinfold thickness: measurements on 481 men and women aged from 16 to 72 years. Br J Nutr 1974; 32: 77-97.

14 Zapletal A, Samanek M, Paul T. Lung Function in Children and Adolescents. Basel, Karger, 1987.

15 Quanjer PH, Tammeling GJ, Cotes JE, et al. Lung volumes and forced ventilatory flows. Report Working Party Standardization of Lung Function Tests, European Community for Steel and Coal. Official Statement of the European Respiratory Society. Eur Respir J 1993; 6: Suppl. 16, 5-40.

16 Wenninger K, Aussage P, Wahn U, et al. The revised German Cystic Fibrosis Questionnaire: validation of a disease-specific healthrelated quality of life instrument. Qual Life Res 2003; 12: 77-85.

17 Hebestreit H, Staschen B, Hebestreit A. Ventilatory threshold: a useful method to determine aerobic fitness in children? Med Sci Sports Exerc 2000; 32: 1964-1969.

18 Orenstein DM, Hovell MF, Mulvihill M, et al. Strength vs aerobic training in children with cystic fibrosis. A randomized controlled trial. Chest 2004; 126: 1204-1214.

19 Hayllar KM, Williams SGJ, Wise AE, et al. A prognostic model for the prediction of survival in CF. Thorax 1997; 52: 313-317.

20 Johansen HK, Hoiby N. Seasonal onset of initial colonisation and chronic infection with Pseudomonas aeruginosa in patients with cystic fibrosis in Denmark. Thorax 1992; 47: 109-111.

21 Sharma R, Florea VG, Bolger AP, et al. Wasting as an independent predictor of mortality in patients with cystic fibrosis. Thorax 2001; 56: 746-750

22 Lieberman DA, Maxwell LC, Faulkner JA. Adaptation of guinea pig diaphragm muscle to aging and endurance training. $A m \mathrm{~J}$ Physiol 1972; 222: 556-560.

23 Powers SK, Lawler J, Criswell D, et al. Endurance-training-induced cellular adaptations in respiratory muscles. J Appl Physiol 1990; 68 2114-2118.

24 Mutluay FK, Demir R, Ozyilmaz S, et al. Breathing-enhanced upper extremity exercises for patients with multiple sclerosis. Clin Rehabil 2007; 21: 595-602.

25 Enright S, Chatham K, Ionescu AA, et al. Inspiratory muscle training improves lung function and exercise capacity in adults with cystic fibrosis. Chest 2004; 126: 405-411.

26 Boas SR. Exercise recommendations for individuals with cystic fibrosis. Sports Med 1997; 24: 17-37.

27 Ruf K, Hebestreit H. Exercise-induced hypoxemia and cardiac arrhythmia in cystic fibrosis. J Cystic Fibros 2009; 8: 83-90.

28 Steinkamp G, Wiedemann B. Relationship between nutritional status and lung function in cystic fibrosis: cross sectional and longitudinal analyses from the German CF quality assurance (CFQA) project. Thorax 2002; 57: 596-601. 